\title{
Unstructured Tetrahedral Mesh Generation Technology
}

\author{
Alexander A. Danilov \\ Institute of Numerical Mathematics \\ Russian Academy of Sciences, Moscow \\ danilov@dodo.inm.ras.ru
}

Key Words: Unstructured Tetrahedral Mesh Generation, Advancing Front Technique, Integration with CAD, Surface Mesh Refinement, Solid Constructive Geometry.

\begin{abstract}
The whole process of mesh generation is presented in detail. Advancing front technique (AFT) is proposed for 3D tetrahedral mesh generation [1]. AFT starts from initial front. Initial front is a boundary surface conformal triangulation. On each step a new tetrahedron is constructed and the front is updated. At this point local mesh size could be selected automatically in order to create quasi-uniform mesh, or mesh coarsened to the interior of the region. Local mesh size could also be controlled by the user explicitly.

AFT method has some issues in 3D case. There are such front configurations in which new tetrahedron can not be created. Another Delaunay-based mesh generation technique is used in this case [2]. This method consists of Delaunay mesh generation followed by boundary faces restoration. This approach allows us to generate tetrahedral meshes starting from initial front fully automatically and still in controlled way.
\end{abstract}

Initial front could be constructed in several ways. Four approaches will be discussed in detail.

1. Analytical boundary parametrisation based on boundary representation (BREP) model.

2. Interface with CAD systems based on the same BREP model.

3. Initial surface mesh refinement.

4. Surface meshing of solid constructive geometry (SCG) models.

Each of these approaches is more suitable for particular cases. For example analytical boundary parametrisation method is more convenient for simple regions like spheres, boxes, and other polyhedrons with planar faces. Complex regions like union of several simple primitives could be processed with solid constructive geometry approach. More complex regions designed in CAD systems could be meshed with the help of interface between CAD and AFT mesh generator. Regions could also be defined by their boundary discretization. Latter may have a bad shape surface triangulation, and thus surface mesh refinement process might be very useful.

Several examples will be presented introducing mesh generation library from Ani3D project [3].

\section{REFERENCES}

[1] George P.L. Automatic mesh generation and finite element computation. Handbook of Num.Anal., 1996, V.4, p.127-148.

[2] George P.L., Borouchaki H., Saltel E. Ultimate robustness in meshing an arbitrary polyhedron. Int. J. Numer. Meth. Eng, 2003, 58, 7, p.1061-1089.

[3] 3D Generator of Anisotropic Meshes. http://sourceforge.net/projects/ani3d/ 